\title{
Floer homology on the universal cover, Audin's conjecture and other constraints on Lagrangian submanifolds
}

\author{
Mihai Damian*
}

\begin{abstract}
We establish a new version of Floer homology for monotone Lagrangian embeddings in symplectic manifolds. As applications, we get assertions for monotone Lagrangian submanifolds $L \hookrightarrow M$ which are displaceable through Hamiltonian isotopies (this happens for instance when $M=\mathbb{C}^{n}$ ). We show that when $L$ is aspherical, or more generally when the homology of its universal cover vanishes in odd degrees, its Maslov number $N_{L}$ equals 2 . This is a generalization of Audin's conjecture. We also give topological characterisations of Lagrangians $L \hookrightarrow M$ with maximal Maslov number: when $N_{L}=\operatorname{dim}(L)+1$ then $L$ is homeomorphic to a sphere; when $N_{L}=n \geq 6$ then $L$ fibers over the circle and the fiber is homeomorphic to a sphere. A consequence is that any exact Lagrangian in $T^{*} S^{2 k+1}$ whose Maslov class is zero is homeomorphic to $S^{2 k+1}$.
\end{abstract}

Mathematics Subject Classification (2010). 57R17, 57R58, 57R70, 53D12.

Keywords. Lagrangian embeddings, Floer homology, Maslov number.

\section{Introduction and main results}

1.1. Preliminaries. Let $\left(M^{2 n}, \omega\right)$ be a symplectic manifold. A submanifold $L^{n}$ of $M$ is called Lagrangian if the restriction of $\omega$ on $L$ vanishes. Throughout this paper all symplectic manifolds are assumed to be either closed or convex at infinity and all Lagrangian submanifolds are assumed to be closed and connected. One of the fundamental questions in symplectic geometry is the following:

What properties has to satisfy a closed manifold $L$ in order to admit a

Lagrangian embedding into a given symplectic manifold $M$ ?

This question is still widely open even in the case of $M=\mathbb{C}^{n}$. The results of the present paper concern symplectic manifolds such as $M=\mathbb{C}^{n}, M=\mathbb{C P}^{n}$, or $M=$ $T^{*} K$ for $K$ closed, all of them being endowed with their standard symplectic form. We establish new topological constraints on Lagrangian submanifolds $L \subset M$ which are monotone or exact. These notions are defined using two morphisms related to a

\footnotetext{
*Supported by ANR project "Floer Power" ANR-08-BLAN-0291-03.
} 
given Lagrangian $L$ which are defined as follows. The morphism $I_{\omega}: \pi_{2}(M, L) \rightarrow \mathbb{R}$ is defined by

$$
I_{\omega}(A)=\int_{A} \omega
$$

In order to define the morphism $I_{\mu}: \pi_{2}(M, L) \rightarrow \mathbb{Z}$, pick a smooth map of pairs $w:\left(D^{2}, \partial D^{2}\right) \rightarrow(M, L)$ in the class $A \in \pi_{2}(M, L)$. There is an unique trivialisation (up to homotopy) of the pull-back $w^{*} T M \approx D^{2} \times \mathbb{C}^{n}$ as a symplectic vector bundle. This gives a map $\alpha_{w}$ from $S^{1}=\partial D^{2}$ to $\Lambda\left(\mathbb{C}^{n}\right)$ - the set of Lagrangian planes in $\mathbb{C}^{n}$. On this space there is a well-known Maslov class $\mu \in H^{1}\left(\Lambda\left(\mathbb{C}^{n}\right), \mathbb{Z}\right)$ (see [2]), so that one can define

$$
I_{\mu}(A)=\mu(\alpha) \in \mathbb{Z}
$$

Definition 1.1. A Lagrangian submanifold $L \subset M$ is called weakly exact if the morphism $I_{\omega}$ vanishes. It is called exact if $\omega=d \lambda$ and the restriction $\left.\lambda\right|_{L}$ is an exact one-form.

A Lagrangian submanifold is called monotone if there is a constant $\tau>0$ such that

$$
I_{\omega}=\tau I_{\mu}
$$

We can see from the definition that only exact symplectic manifolds admit exact Lagrangian submanifolds. It is less obvious, but still true, that monotone Lagrangian submanifolds only exist in monotone symplectic manifolds (i.e. in symplectic manifolds in which the morphism defined on $\pi_{2}(M)$ by the first Chern class is a positive multiple of the morphism defined by the symplectic form). Many authors studied monotone and exact Lagrangians and found various obstructions to the existence of such embeddings. A celebrated result of M. Gromov asserts:

Theorem 1.2 ([26]). There is no weakly exact Lagrangian embedding $L \subset \mathbb{C}^{n}$.

The results on the obstructions to the existence of monotone Lagrangian submanifolds mostly concern their Maslov number. This number, denoted by $N_{L}$, is defined as the positive generator of the image of $I_{\mu}$. In 1996, Y.-G. Oh established the following inequality [34], improving thus previous results of L. Polterovich [36], [37]:

Theorem 1.3. For any monotone Lagrangian submanifold $L \subset \mathbb{C}^{n}$ we have

$$
1 \leq N_{L} \leq n
$$

These bounds turn out to be sharp. Indeed, Polterovich gave in [37] an example of a monotone Lagrangian $L \subset \mathbb{C}^{n}$ which satisfies $N_{L}=n$.

Note that both Gromov's and Oh's result can be stated for the more general case of symplectic manifolds $M$ which are convex at infinity and have the property that any 
compact subset is displaceable through a Hamiltonian isotopy. This means that for any compact $K \subset M$ there is a Hamiltonian isotopy $\left(\phi_{t}\right)_{t \in[0,1]}$ such that $\phi_{1}(K) \cap K=\emptyset$. Symplectic manifolds of the form $\mathbb{C} \times W$, or subcritical Stein manifolds satisfy to this assumption.

However, in this more general case Oh's result is not true as it is stated above. The correct formulation is:

Theorem 1.4. Let $M$ be a symplectic manifold in which every compact subset is displaceable through a Hamiltonian isotopy. For any monotone Lagrangian submanifold $L \subset M$ we have

$$
1 \leq N_{L} \leq n+1
$$

and if $N_{L}=n+1$, then $L$ is a $\mathbb{Z} / 2$-homology sphere.

Actually, more recent results of K. Fukaya, Y.-G. Oh, H. Ohta and K. Ono [22] imply that for $N_{L}=n+1$, the Lagrangian $L$ is a $\mathbb{Z}$-homology sphere in the statement above.

1.2. Main results. Our results about monotone Lagrangian submanifolds are of two types. First we show that under some topological assumptions on $L$ we have $N_{L}=2$. Then, we study the topology of monotone Lagrangian submanifolds with maximal Maslov number $N_{L}=n+1$ or $N_{L}=n$. Here are the statements:

Theorem 1.5 (Audin's conjecture). Let $M$ be a monotone symplectic manifold which has the property that any compact subset is displaceable through a Hamiltonian isotopy. Let $L \subset M$ be a monotone Lagrangian submanifold.

a) If $L$ is aspherical (i.e. $L$ is an Eilenberg-McLane space $K\left(\pi_{1}(L), 1\right)$ ), then $N_{L}=2$ if $L$ is orientable and $N_{L} \in\{1,2\}$ if $L$ is not orientable.

b) Denote by $\widetilde{L}$ the universal cover of $L$. If $L$ is orientable and has the property

$$
H_{2 i+1}(\tilde{L}, \mathbb{Z} / 2)=0
$$

for any integer $i$, then $N_{L}=2$.

c) Moreover, for any almost complex structure $J$ which is compatible with the symplectic form, a Lagrangian $L$ which satisfies to the condition b) has the property that through every $p \in L$ there is a J-holomorphic disk $w:(D, \partial D) \rightarrow(M, L)$ such that:

- The Maslov index $\mu(w)$ equals 2.

- $p \in w(\partial D)$.

- $w(\partial D)$ is non-zero in $\pi_{1}(L)$. 
Remarks. 1. Part a) of Theorem 1.5 was proved by K. Fukaya for general aspherical Lagrangian submanifolds, but under the additional hypothesis that $L$ is orientable and relatively spin ([21], Theorem 12.2). In the case where $L$ is a torus the statement was conjectured by M. Audin [3]. In this particular case, many results were previously obtained by L. Polterovich, C. Viterbo, Y.-G. Oh, Y. Eliashberg, P. Biran, K. Cieliebak, K. Mohnke and L. Buhovsky.

2. Part b) of the statement above was proved by K. Fukaya in the case $L=$ $S^{1} \times S^{2 m}$ without any monotonicity assumption ([21], Theorem 13.1). However, our result applies to many more general examples, such as arbitrary products of tori (or other orientable aspherical manifolds) and complex projective spaces, evendimensional spheres, etc.

3. Many results related to Part c) of the theorem can be found in the paper [11] of P. Biran and O. Cornea. Using their terminology, the Lagrangian $L$ should be called uniruled of type $(0,1)$ and order 2 .

Using the ideas of P. Biran [7], we obtain the following corollary on the monotone Lagrangian submanifolds in the complex projective space.

Theorem 1.6. Let $W$ be a symplectic manifold such that $M=\mathbb{C} \mathbf{P}^{n} \times W$ is monotone (for instance this holds for $\pi_{2}(W)=0$ or for $W=\mathbb{C P}^{n}$ ). Let $L \subset M$ be a monotone Lagrangian submanifold which is aspherical. Then $N_{L}=2$ if $L$ is orientable and $N_{L} \in\{1,2\}$ if $L$ is not orientable.

For spin Lagrangian submanifolds and $W=$ point this result was also proved by K. Fukaya in [21] without any monotonicity assumption. The result is still true in the more general situation where $\mathbb{C} \mathbf{P}^{n}$ is replaced by a symplectic manifold which arises as a hypersurface in a subcritical polarisation. These manifolds were studied in [8] by P. Biran and K. Cieliebak.

Our next result is a topological characterisation of monotone Lagrangian submanifolds with maximal Maslov number.

Theorem 1.7. Let $M$ be a (monotone) symplectic manifold of dimension $2 n$, which has the property that any compact subset is displaceable through a Hamiltonian isotopy. Let $L \subset M$ be a monotone Lagrangian submanifold.

a) Suppose that $N_{L}=n+1$ and $n \geq 2$. Then $n$ is odd and $L$ is homeomorphic to the $n$-sphere.

b) Suppose that $N_{L}=n$ and $n \geq 3$.

If $n$ is odd then $\pi_{1}(L)$ has an infinite cyclic group $G \approx \mathbb{Z}$ of finite index. If moreover $M$ is an exact symplectic manifold then there is an exact sequence of groups

$$
0 \rightarrow K \rightarrow \pi_{1}(L) \rightarrow \mathbb{Z} \rightarrow 0,
$$

where $K$ is finite and has odd order. 
If $n$ is even then $\pi_{1}(L) \approx \mathbb{Z}$. If moreover $n \geq 6$, then there is a fibration of $L$ over the circle $S^{1}$ whose fiber is homeomorphic to the $(n-1)$-sphere.

Remarks. 1. There are examples of monotone Lagrangian submanifolds satisfying the hypothesis on the Maslov number above. Indeed the embedding of $S^{2 k+1}$ into $\mathbb{C} \mathbf{P}^{k} \times \mathbb{C}^{k+1}$ given by

$$
z \mapsto([z], \bar{z})
$$

is monotone, Lagrangian and its Maslov number is $2 k+2$. This example is due to M. Audin, F. Lalonde and L. Polterovich [5]. An example of a monotone Lagrangian embedding $S^{1} \times S^{2 k-1} \subset \mathbb{C}^{2 k}$ whose Maslov number equals $2 k$ was constructed by L. Polterovich in [37].

2. In [25] A. Gadbled established topological constrains on monotone Lagrangian submanifolds in cotangent bundles which have a large Maslov number (which implies that they are not displaceable through Hamiltonian isotopies).

We prove the following corollaries of this theorem:

Theorem 1.8. a) Let $X$ be a symplectic manifold of dimension $2 n+2$ with $\pi_{2}(X)=0$. Let $L \subset \mathbb{C P}^{n} \times X$ be a Lagrangian submanifold such that $H_{1}(L, \mathbb{Z})=0$. Then $L$ is homeomorphic to $S^{2 n+1}$.

b) Let $L \subset \mathbb{C} \mathbf{P}^{n} \times \mathbb{C} \mathbf{P}^{n}$ be a Lagrangian submanifold such that $H_{1}(L, \mathbb{Z})$ vanishes. Then $L$ is simply connected and there is a circle fibration $S^{2 n+1} \rightarrow L$.

c) Let $L \subset \mathbb{C P}^{n}$ be a Lagragian submanifold such that $2 x=0$ for any $x \in$ $H^{1}(L, \mathbb{Z})$. Then if $n$ is odd we have $\pi_{1}(L)=\mathbb{Z} / 2$ and the universal cover of $L$ is homeomorphic to $S^{n}$.

In [9] O. Cornea and P. Biran asked whether a Lagrangian as in 1.8.c is diffeomorphic (or homeomorphic) to $\mathbb{R} \mathbf{P}^{n}$. Our result goes in this direction but we do not know whether its conclusion implies that $L$ is homeomorphic to the projective space. In the mentioned paper Biran and Cornea proved that under the hypothesis of 1.8 the cohomology ring (with $\mathbb{Z} / 2$-coefficients) of $L$ is isomorphic to the cohomology ring of $\mathbb{R} \mathbf{P}^{n}$. Similar results were previously obtained by P. Biran [7] and P. Seidel [38]. The statement a) generalizes Theorem B of [7] (asserting that $L$ is a homology sphere). The statement b) generalizes Theorem $\mathrm{C}$ of [7] (which asserts that $L$ has the homology of $\mathbb{C} \mathbf{P}^{n}$ ).

Theorem 1.9. a) Let $L \subset T^{*} S^{2 k+1}$ be an exact Lagragian submanifold with vanishing Maslov class. Then $L$ is homeomorphic to $S^{2 k+1}$.

b) Let $K^{2 k+1}$ a manifold whose universal cover is $S^{2 k+1}$. Let $L \subset T^{*} K$ be an exact Lagragian submanifold with vanishing Maslov class. Then the universal cover $\widetilde{L}$ is homeomorphic to $S^{2 k+1}$ (in particular $\pi_{1}(L)$ is finite). For instance, when $K=\mathbb{R} \mathbf{P}^{2 k+1}$ then $\pi_{1}(L)=\mathbb{Z} / 2 Z$ and $L$ is double covered by (a manifold homeomorphic to) $S^{2 k+1}$. 
This result gives an answer in the case $K=S^{2 k+1}$ (under the hypothesis of the vanishing Maslov class) to an open question raised by V. I. Arnold [1]:

Is an exact submanifold $L \subset T^{*} K$ homeomorphic to $K$ ?

Actually, Arnold asks whether $L$ is Hamiltonian isotopic to the zero section, but this latter question seems out of reach, except for the case $\operatorname{dim}(K)=2$ (see [27] for related results). In the general case the most striking result was obtained by K. Fukaya, P. Seidel and I. Smith, who proved in [23], [24] that when $L$ is relatively spin with vanishing Maslov class, its cohomology is isomorphic to the cohomology of $K$. For the case $K=S^{m}$ similar results were previously obtained by P. Seidel [38] and L. Buhovsky [12].

1.3. Idea of the proofs. The proofs are based on a new version of Floer homology which is constructed using an arbitrary covering of the Lagrangian submanifold. In order to present it we need to recall some facts about the original Floer homology construction.

1.3.1. The original Floer setting. Let $L \subset M$ be a Lagrangian submanifold which is monotone with $N_{L} \geq 2$, or weakly exact. Consider a Hamiltonian isotopy $\left(\phi_{t}\right)$ defined by a time-dependent Hamiltonian $H:[0,1] \times M \rightarrow \mathbb{R}$ and an almost complex structure $J$ which is compatible with the symplectic form $\omega$. For a generic choice of the couple $(H, J)$, A. Floer associated to these data a complex $\left(C_{\bullet}(H), \partial_{J}\right)$ whose homology does not depend on $(H, J)$ [17], [18], [19]. The Floer complex is free over $\mathbb{Z} / 2$, spanned by the intersections $L \cap \phi_{1}(L)$, which are supposed to be transverse. Its differential $\partial_{J}$ is defined by counting the isolated holomorphic strips

$$
w: \mathbb{R} \times[0,1] \rightarrow M,
$$

with boundary in $L \cup \phi_{1}(L)$ (more precisely $w(\mathbb{R} \times\{i\}) \subset \phi_{i}(L)$ for $\left.i=0,1\right)$ and joining intersection points $x, y \in L \cap \phi_{1}(L)$, which means that

$$
\lim _{s \rightarrow-\infty} w(s, t)=x \text { and } \lim _{s \rightarrow+\infty} w(s, t)=y .
$$

If $n(x, y)$ is the number modulo 2 of such curves then

$$
\partial_{J}(x)=\sum_{y \in L \cap \phi_{1}(L)} n(x, y) y .
$$

Note that if $L$ is orientable and relatively spin (meaning that the second StiefelWhitney class $w_{2}(L)$ lies in the image of $\left.H^{2}(M, \mathbb{Z} / 2) \rightarrow H^{2}(L, \mathbb{Z} / 2)\right)$, then the whole theory works for integer coefficients [22].

A relation between the Floer homology $H F(L)$ and the usual homology can be established. At this end, one should remark that given a Morse function $f: L \rightarrow \mathbb{R}$ which is sufficiently $\ell^{1}$-small, its graph

$$
\left\{\left(d f_{q}, q\right) \mid q \in L\right\} \subset T^{*} L
$$


can be embedded in $M$ via a Weinstein neighborhood $U(L) \subset M$ and the intersection points $L \cap L_{f}$ correspond to the critical points of $f$. Moreover, for a good choice of the almost complex structure $J$, the application

$$
w(s, t) \mapsto w(s, 0)
$$

defines a one-to-one correspondence between the holomorphic strips joining two intersection points $x, y$ which lie in $U(L)$ and the flow lines of a vector field on $M$ which is the gradient of $f$ with respect to some Riemannian metric. So the Morse complex becomes a sub-complex of the Floer complex in this case. If $L$ is weakly exact, and $f$ is chosen sufficiently small, one can prove that no holomorphic strip leaves $U(L)$, so that the two complexes are actually isomorphic and thus Floer homology is isomorphic to usual homology. In the case where $L$ is monotone, Y.-G. Oh shows in [34] that in the case of the particular Hamiltonian isotopy defined by the graph of a small function $f$, the Floer differential decomposes into a sum

$$
\partial_{J}=\partial_{0}+\partial_{1}+\partial_{2}+\cdots,
$$

where $\partial_{0}: C_{k}(f) \rightarrow C_{k-1}(f)$ is the Morse differential and $\partial_{l}: C_{k}(f) \rightarrow C_{k-1+l N_{L}}$ for any integer $l$. By comparing the degrees of the $\partial_{i}$ 's in the relation $\partial_{J}^{2}=0$ one easily sees that $\partial_{1}$ defines an application of degree $-1+N_{L}$ on the usual homology groups of $L$ and moreover that this application is actually a differential. On the resulting homology groups $\partial_{2}$ defines an application of degree $-1+2 N_{L}$ which again turns out to satisfy $\partial_{2}^{2}=0$, and so on... This feature of the Floer differential can be formalized in the existence of a spectral sequence which converges to the Floer homology $\operatorname{HF}(L)$ and whose first page is built using the usual cohomology groups of $L$ [34], [7].

1.3.2. The difference between the cases $N_{L}=2$ and $N_{L} \geq 3$. As said above, the Lagrangian Floer homology $H F(L)$ can be defined for monotone Lagrangian submanifolds with Maslov number $N_{L} \geq 2$. But the proof of this fact is slightly different in the case $N_{L}=2$. Since this difference is important in the sequel, we will explain it below, using the simple example of an embedded circle in $\mathbb{C}$.

The fact that $\partial_{J}$ defines a complex is equivalent (with the notation above) to

$$
\sum_{y \in L \cap \phi_{1}(L)} n(x, y) n(y, z)=0 \bmod 2,
$$

for any $x, z \in L \cap \phi_{1}(L)$. The usual proof of this assertion in Morse theory requires to show that any broken trajectory which joins $x$ and $z$ via another intersection point $y$ is a boundary point in a 1-dimensional manifold whose open part is a 1-dimensional component of the space of trajectories between $x$ and $z$. This implies that the total number of broken trajectories is even and therefore the above relation is satisfied and the Floer complex is well defined. 
In the case of a monotone Lagrangian submanifold $L$, this proof works for $N_{L} \geq 3$ (see Theorem 2.3 and Theorem 2.4 below). But it fails for $N_{L}=2$. To illustrate this, let us consider the intersection between an embedded circle $L \subset \mathbb{C}$ and its image by a translation as in Figure 1 below.

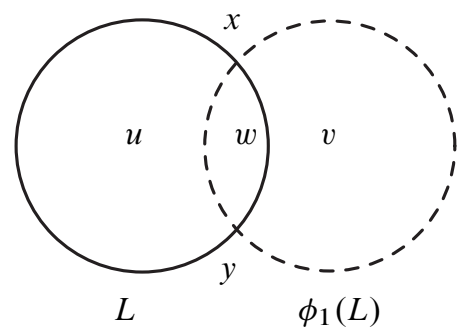

Figure 1 . The case $N_{L}=2$.

As one can easily see, the Maslov number $N_{L}$ is indeed equal to 2 . The differential (associated to the standard complex structure) is given by the three holomorphic strips, denoted by $u, v$ and $w$. More precisely,

$$
\partial(x)=u \cdot y+v \cdot y=0 \quad \text { and } \quad \partial(y)=w \cdot x=x,
$$

where the notation $u \cdot x(=x)$ is used to emphasize that the coefficient of $x$ is given by the strip $u$. So, $\partial^{2}=0$ and the complex is defined. But there is no 1-manifold consisting of holomorphic strips which have the broken orbits $u \# w$ and $v \# w$ as boundary points. As we see in Figure 2 below, there is a path of holomorphic strips $\Delta_{t}$ passing through $x$, whose ends are on one side the broken orbit $u \# v$ and on the other side the holomorphic disk $D$ with boundary in $L$. Analogously, the broken orbit $v \# w$ is connected through holomorphic strips to the holomorphic disk with boundary in $\phi_{1}(L)$.
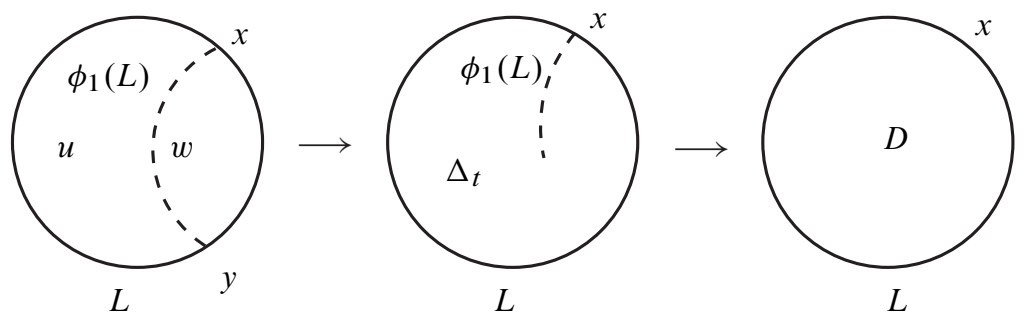

Figure 2. Broken orbits and holomorphic disks.

The reason why the Floer complex is still well defined here is that there are exactly two holomorphic disks with boundary either in $L$ or in $\phi_{1}(L)$ and therefore 
the number of broken orbits is even. This is a particular case of a more general phenomenon which occurs when $N_{L}=2$. Namely the number of holomorphic disks passing through a intersection point $x \in L \cap \phi_{1}(L)$ having the boundary contained in one of these submanifolds is even. And any such disk can be connected either to another similar disk or to a broken orbit joining $x$ to itself. This result, due to Y.-G. Oh [33] (see Theorem 3.2 below), enables us to define the Floer homology $H F(L)$ when $N_{L}=2$. The relation between Floer and usual homology is the same as for $N_{L} \geq 3$.

1.3.3. Floer homology on a covering space. The idea of our proofs is the following. Fix a covering space $\bar{L} \rightarrow L$. Given a Morse function on $L$ and an associated generic gradient, one can build a free complex, possibly infinite-dimensional, by lifting its flow lines to $\bar{L}$. The homology of this complex is the usual (singular) homology $H_{*}(\bar{L})$.

Now consider the Floer complex associated to $L$ and to some generic pair $(H, J)$. Look at the collection of paths $\gamma_{w}$ defined by $s \mapsto w(s, 0) \subset L$ of holomorphic strips $w: \mathbb{R} \times[0,1] \rightarrow M$ which define the Floer differential $\partial_{J}$. Obviously the Floer complex can be reconstructed from the collection of these paths, by counting the number of those who have the same endpoints $x$ and $y$ and defining thus a differential on the free module spanned by all these endpoints. On the other hand one can lift these paths to the covering space $\bar{L}$. The question is:

Do the lifted paths define a complex ?

This turns out to be true when $L$ is weakly exact or monotone with $N_{L} \geq 3$ (see Proposition 2.6 below). But it fails for $N_{L}=2$, as one can easily see by looking again at the example of an embedded circle in $\mathbb{C}$ which we considered in the previous subsection. We draw in Figure 3 below the paths $\gamma$ which define the new differential. For the trivial covering $L \rightarrow L$ we get of course the same thing, namely

$$
\partial(x)=\gamma_{u} \cdot y+\gamma_{v} \cdot y \quad \text { and } \quad \partial(y)=\gamma_{w} x,
$$

so that $\partial^{2}=0$. But when we consider the universal covering for instance, we get

$$
\partial^{2}(x)=\left(\gamma_{u} \# \gamma_{w}\right) \cdot x+\left(\gamma_{v} \# \gamma_{w}\right) \cdot x \neq 0
$$

since the paths $\gamma_{u} \# \gamma_{w}$ and $\gamma_{v} \# \gamma_{w}$ are not homotopic in $L$. Therefore, we do not get a complex in this case. This can be explained by the fact that, unlike in the usual case, the holomorphic disk with boundary in $\phi_{1}(L)$ does not have any contribution to the differential, as long as we only look at the submanifold $L$. Let us go back to the case where $L$ is weakly exact, or monotone with $N_{L} \geq 3$, when our complex is defined. In the particular case of the Hamiltonian isotopy defined by a graph $L_{f}$ of a small function, the differential $\partial_{0}$ clearly defines the lifted Morse complex described at the beginning of the present subsection. Therefore, when this lifted Floer complex is defined, its homology has analogue features, namely it coincides with the (Morse) 


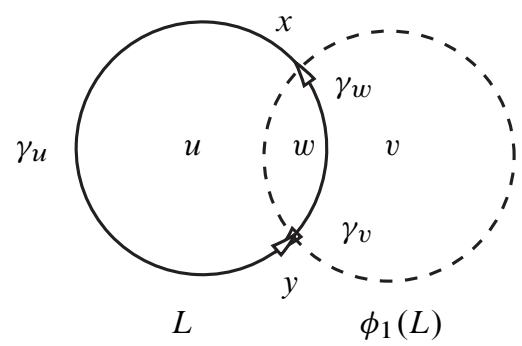

Figure 3. Definition of the lifted complex.

homology of the covering $\bar{L}$ if $L$ is weakly exact and it is the limit of a spectral sequence starting from the homology of $\bar{L}$ when $L$ is monotone. This allows us to infer the claimed results, by using the homology of the universal cover. Sometimes, as for instance in the case of a monotone aspherical Lagrangian, the existence of the lifted complex leads to a contradiction, which implies $N_{L} \leq 2$, proving Audin's conjecture 1.5.a).

The construction of the new complex will be formalized in the next section.

\section{The lifted Floer complex}

Let $L \subset M$ be a Lagrangian submanifold. Let $p: \bar{L} \rightarrow L$ be a covering of $L$. The elements of a fiber of $p$ are indexed by a possibly infinite set $I$. Let $\left(\phi_{t}\right)_{t \in[0,1]}$ be a Hamiltonian isotopy of $M$ such that $L$ and $\phi_{1}(L)$ are transverse. For any $x \in L \cap \phi_{1}(L)$ denote by $\left(x_{i}\right)_{i \in I}$ the elements of $p^{-1}(x)$. We prove the following theorem, which is the main ingredient in the proof of the results that we claimed in the preceding section:

Theorem 2.1. If $L$ is exact or monotone with $N_{L} \geq 3$, there exist a free $\mathbb{Z} / 2$-complex C. spanned by $\bigcup_{x \in L \cap \phi_{1}(L)}\left\{x_{i} \mid i \in I\right\}$ such that:

- If $L$ is exact then

$$
H_{*}\left(C_{\bullet}\right) \approx H_{*}(\bar{L}, \mathbb{Z} / 2)
$$

- If $L$ is monotone with $N_{L} \geq 3$ then there exist applications $\delta_{1}, \delta_{2}, \ldots, \delta_{k}, \ldots$ with the following properties:

- $\delta_{1}: H_{*}(\bar{L}, \mathbb{Z} / 2) \rightarrow H_{*-1+N_{L}}(\bar{L}, \mathbb{Z} / 2)$.

- $\delta_{l}: H_{*}(\bar{L}, \mathbb{Z} / 2) \rightarrow H_{*-1+l N_{L}}(\bar{L}, \mathbb{Z} / 2)$ is well defined if $\delta_{m}=0$ for $m=1, \ldots, l-1$. 
- If $\delta_{l}=0$ for any $l \geq 1$ then

$$
H_{*}\left(C_{\bullet}\right) \approx H_{*}(\bar{L}, \mathbb{Z} / 2)
$$

If $\bar{L}=\tilde{L}$ is the universal cover of $L$ then $C_{\bullet}$ can be viewed as a free, finitedimensional complex over $\mathbb{Z} / 2\left[\pi_{1}(L)\right]$, spanned by $\phi_{1}(L) \cap L$. A $C W$-decompostion of $L$ can be lifted to $\widetilde{L}$ and gives rise to a free $\mathbb{Z} / 2\left[\pi_{1}(L)\right]$-complex, $D_{\bullet}$. Then, given $a \mathbb{Z} / 2\left[\pi_{1}(L)\right]$-module $P$, the statements above are valid when one replaces $C \bullet$ by $C \bullet \otimes_{\mathbb{Z} / 2\left[\pi_{1}(L)\right]} P$ and $H_{*}(\tilde{L})$ with the homology of the complex $D \bullet \otimes_{\mathbb{Z} / 2\left[\pi_{1}(L)\right]} P$. For instance, when $P$ is the Novikov ring associated to some 1-cohomology class $u$, the latter is the Novikov homology $H_{*}(L ; u)$. More generally, the analogous assertion for coverings $\bar{L} \rightarrow L$ associated to normal subgroups $G \leq \pi_{1}(L)$ is also valid.

When $L$ is orientable and relatively spin, one can replace $\mathbb{Z} / 2$-coefficients with $\mathbb{Z}$-coefficients in all the statements of this theorem.

Proof. 1. The usual Floer complex. Let us start by reminding the definition of the original Floer complex $F C_{\bullet}\left(L,\left(\phi_{t}\right)\right)$ over $\mathbb{Z} / 2$. It is spanned by the intersection points $L \cap \phi_{1}(L)$. Denote $L_{t}=\phi_{t}(L)$. In order to define the differential of $F C_{\bullet}$, one has to choose an almost complex structure $J$ on $M$ which is compatible with the symplectic form $\omega$ and to define the space of holomorphic strips with bounded energy $\mathcal{M}\left(L_{0}, L_{1}\right)$, as follows:

$$
\mathcal{M}\left(L_{0}, L_{1}\right)=\left\{\begin{array}{l|l}
v \in \mathcal{C}^{\infty}(\mathbb{R} \times[0,1], M) & \begin{array}{l}
\frac{\partial v}{\partial s}+J \frac{\partial v}{\partial t}=0, \\
v(s, 0) \in L_{0}, v(s, 1) \in L_{1}, \\
E(v)<+\infty
\end{array}
\end{array} .\right.
$$

Here, the energy $E(v)$ is defined by the formula

$$
E(v)=\int_{\mathbb{R} \times[0,1]}\left\|\frac{\partial v}{\partial s}\right\|^{2} d s d t,
$$

the norm being defined by the Riemannian metric $\omega(\cdot, J \cdot)$. Then consider for $x, y \in$ $L_{0} \cap L_{1}$ the space

$$
\mathcal{M}(x, y)=\left\{v \in \mathcal{M}\left(L_{0}, L_{1}\right) \mid \lim _{s \rightarrow-\infty} v(s, \cdot)=x \text { and } \lim _{s \rightarrow+\infty} v(s, \cdot)=y\right\} .
$$

Floer proved in [17], [19] the following:

Theorem 2.2. a) We have

$$
\mathcal{M}\left(L_{0}, L_{1}\right)=\bigcup_{x, y \in L_{0} \cap L_{1}} \mathcal{M}(x, y) .
$$

b) For a generic choice of $J$ the spaces $\mathcal{M}(x, y)$ are finite-dimensional manifolds of local dimension $\mu(v)=$ the Maslov-Viterbo index of $v$ (see [39] for the definition). 
Denote by $\mathscr{L}^{0}(x, y)$ the 0 -dimensional component of $\mathscr{L}(x, y)=\mathcal{M}(x, y) / \mathbb{R}$. To define the differential of $F C$. we need to prove that $\mathscr{L}^{0}(x, y)$ is finite. This is a consequence of Gromov's compactness for holomorphic curves [26] and was proved by Floer in [17] and Y.-G. Oh in [32]:

Theorem 2.3. Suppose that $L$ is compact, either weakly exact, or monotone with $N_{L} \geq 3$. Let $x, y \in L_{0} \cap L_{1}$ and $A>0$ and let $\left.\left(v_{n}\right) \subset \mathcal{M}_{(} x, y\right)$ be a sequence of solutions with constant index $\mu\left(v_{n}\right)=\mu_{0} \leq 2$. Then there exist a finite collection $\left(z_{i}\right)_{i=0, \ldots, k}$ of points in $L_{0} \cap L_{1}$ with $z_{0}=x$ and $z_{k}=y$, some solutions $v^{i} \in$ $\mathcal{M}_{A}\left(z_{i-1}, z_{i}\right)$ for $i=1, \ldots, k$ and some sequences of real numbers $\left(\sigma_{n}^{i}\right)_{n}$ for $i=$ $1, \ldots, k$ such that for all $i=1, \ldots, k$ the sequence $v_{n}\left(s+\sigma_{n}^{i}, t\right)$ converges towards $v^{i}(s, t)$ in $\bigodot_{\mathrm{loc}}^{\infty}$.

Moreover, we have the relation

$$
\sum_{i=1}^{k} \mu\left(v^{i}\right)=\mu_{0}
$$

If $\mu_{0}=1$ in the statement above then necessarily $k=1$ due to the latter relation, so we immediately infer that the spaces $\mathscr{L}^{0}(x, y)$ are compact, which implies that they are finite. This enables one to define the differential $\partial: F C_{\bullet} \rightarrow F C_{\bullet}$ as

$$
\partial(x)=\sum_{y \in L_{0} \cap L_{1}} n(x, y) y,
$$

where $n(x, y)=\# \mathscr{L}^{0}(x, y) \bmod (2)$. In order to prove the relation $\partial^{2}=0$, one has to study the compactness of the 1-dimensional component $\mathscr{L}^{1}(x, y)$ of $\mathscr{L}(x, y)$. Using again 2.3 we find:

Theorem 2.4. Denote by $\overline{\mathscr{L}}^{1}(x, y)$ the union

$$
\mathscr{L}^{1}(x, y) \cup \bigcup_{z \in L_{0} \cap L_{1}} \mathscr{L}^{0}(x, z) \times \mathscr{L}^{0}(z, y),
$$

endowed with the topology given by the convergence towards broken orbits which was defined in 2.3 .

Then $\overline{\mathscr{L}}^{1}(x, y)$ is a compact 1-dimensional manifold whose boundary is

$$
\bigcup_{z \in L_{0} \cap L_{1}} \mathscr{L}^{0}(x, z) \times \mathscr{L}^{0}(z, y) .
$$

Note that the proof of the fact that $\overline{\mathscr{L}}^{1}(x, y)$ is a manifold with boundary requires a gluing argument as in [18]. Now the fact that a compact 1-dimensional manifold has a boundary of even cardinality immediately implies $\partial^{2}=0$, proving thus that $\left(F C_{\bullet}, \partial\right)$ is a complex. 
Remark 2.5. In the monotone case the hypothesis $N_{L} \geq 3$ is crucial in Theorem 2.4 and in Theorem 2.3. The reason is that for $N_{L} \geq 3$ no bubbling holomorphic disk can occur in a sequence of elements of the 2-dimensional component of $\mathcal{M}(x, y)$ and this implies the existence of a subsequence which converges towards a broken orbit, via Gromov compactness.

As explained in Section 1.3.2, Theorem 2.4 is no longer true when $N_{L}=2$. Usual Floer homology is still defined in this case whereas lifted Floer homology is not (see again the explanations in $§ 1.3 .2$ ).

We present below the definition of the lifted Floer homology for Lagrangians $L$ which are weakly exact or monotone with $N_{L} \geq 3$. A sufficient condition to extend this definition to the case of monotone Lagrangians with $N_{L}=2$ is presented in the proof of 1.5.c below.

2. The lifted Floer complex. A construction of a lifted Floer-type complex was already sketched in our previous work [15]. The idea of constructing such a complex was suggested in [6].

Consider the intersection points $L \cap \phi_{1}(L)$, viewed as points in $L$. For two such points $x, y$, any holomorphic strip $v \in \mathcal{M}(x, y)$ defines a path $\gamma:]-\infty,+\infty[\rightarrow L$ which joins $x$ and $y$ :

$$
\gamma(s)=v(s, 0) \text {. }
$$

We consider the obvious extension of $\gamma$ to $[-\infty,+\infty]$ keeping the same notation for the extended path. Look at the collection of intersection points and take the paths $\gamma$ as above, defined by the strips $v$ which belong to the 1-dimensional components of $\mathcal{M}(x, y)$ (which correspond to the 0 -dimensional components of $\mathscr{L}(x, y)$ ). This collection of points and paths joining them is obviously sufficient to re-construct the complex $F C_{\bullet}$. Denote by $\ell$ the collection of points and by $\Gamma$ the collection of paths.

Now start with the above collection of points and paths $(\mathcal{C}, \Gamma)$ and fix a covering $p: \bar{L} \rightarrow L$. For any point $x \in \mathcal{C}$ denote by $\left(x_{i}\right)_{i \in I}$ the elements of the fiber $p^{-1}(x)$. Consider all the lifts of the paths of $\Gamma$ to the covering space $\bar{L}$. It is clear that for fixed points $x_{i}, y_{j}$ (where $\left.i, j \in I\right)$, the lifted space $\mathscr{L}^{0}\left(x_{i}, y_{j}\right)$ is finite; let $n\left(x_{i}, y_{j}\right)$ be its parity. On the free $\mathbb{Z} / 2$-complex $C_{\bullet}^{\bar{L}}$ spanned by $\bigcup_{x \in L \cap \phi_{1}(L)} p^{-1}(x)$ one can therefore define an application $\partial^{\bar{L}}: C_{\bullet}^{\bar{L}} \rightarrow C_{\bullet}^{\bar{L}}$ by the formula

$$
\partial^{\bar{L}}\left(x_{i}\right)=\sum_{p\left(y_{j}\right)=y \in \mathcal{C}} n\left(x_{i}, y_{j}\right) y_{j} .
$$

The sum above is obviously finite since $\Gamma$ is finite and any path $\gamma \in \Gamma$ admits only one lifting starting from $x_{i}$. We prove

Proposition 2.6. $\left(C_{\bullet}^{\bar{L}}, \partial^{\bar{L}}\right)$ is a complex. 
Proof. This is equivalent to the fact that, given $x_{i}, y_{j}$, there is an even number of "broken paths" joining them. Broken paths means lifts of concatenations $\gamma_{1} * \gamma_{2}$, where $\gamma_{i} \in \Gamma$ and $\gamma_{1}$ starts from $x$ and ends into some point $z \in \mathcal{C}$, while $\gamma_{2}$ starts from the same point $z$ and ends in $y$.

Since the paths of $\Gamma$ define a complex (namely the Floer complex $F C_{\bullet}$ ), we know that the number of broken paths joining $x$ and $y$ downstairs is even. Moreover, they represent boundary points of a 1-dimensional compact manifold, so they can naturally be dispatched in a disjoined union of sets of two elements, corresponding to the boundary points of each component of the mentioned 1-dimensional manifold. But in order to get the same property at the level of the covering space $\bar{L}$ one has to check that the broken paths in such a set admit liftings with the same endpoints:

Proposition 2.7. Let $\left\{\gamma_{1} * \gamma_{2}, \gamma_{1}^{\prime} * \gamma_{2}^{\prime}\right\}$ be a set of two broken paths in $L$ as above. Then these broken paths are homotopic in $L$.

Proof. The concatenations $\gamma_{1} * \gamma_{2}$ and $\gamma_{1}^{\prime} * \gamma_{2}^{\prime}$ have the same starting point $x \in \mathcal{C}$ and the same ending point $y \in \mathcal{C}$. We use the following lemma which was proved in [15] (Lemma 3.16).

Lemma 2.8. Let $\left(v_{n}\right)_{n \in \mathbf{N}}$ be a sequence in $\mathcal{M}(x, y)$, as in 2.3. Let $\gamma_{n}:[-\infty,+\infty] \rightarrow$ $L_{0}$ be the path defined by $\gamma_{n}(s)=v_{n}(s, 0)$ extended by $x$ in $s=-\infty$ and by $y$ in $s=+\infty$. For $i=1, \ldots, k$ let $\gamma^{i}:[-\infty,+\infty] \rightarrow L_{0}$ be the analogous paths defined by the holomorphic strips $v^{i}$. Then for $n$ large enough $\gamma_{n}$ and $\gamma=\gamma^{1} * \gamma^{2} * \cdots * \gamma^{k}$ are homotopic in $L_{0}$.

Now the broken holomorphic strip $\left(v^{1}, v^{2}\right)$ which defines $\gamma_{1} * \gamma_{2}$ corresponds to a boundary point in $\overline{\mathscr{L}}^{1}(x, y)$, which means that it is the limit of a sequence lying in a 2-dimensional component of $\mathcal{M}(x, y)$. The same is true for the broken path $\gamma_{1}^{\prime} * \gamma_{2}^{\prime}$. Using the previous lemma we infer that there is some component of $\mathscr{L}^{1}(x, y)$ such $\gamma_{1} * \gamma_{2}$ and $\gamma_{1}^{\prime} * \gamma_{2}^{\prime}$ are respectively homotopic to paths in $L_{0}$ defined by some elements in this component. On the other hand all paths defined by the elements of the same component of $\mathscr{L}^{1}(x, y)$ are obviously homotopic, which finishes the proof of our claim 2.7.

The proof of 2.6 immediately follows, since the previous proposition implies that the set of paths in $\Gamma$ whose lifts in $\bar{L}$ join some fixed points $x_{i}, y_{j}$ is a disjoined union of sets with two elements.

3. Hamiltonian invariance. The usual way (see [18], [32]) to prove that the homology of the Floer complex $F C$. does not depend of the (generic) choice of the Hamiltonian and of the almost complex structure, is to consider a generic homotopy $\Psi_{s}=\left(H_{s, t}, J_{s}\right)$ between two fixed couples $\left(H_{t}, J_{t}\right)$ and $\left(H_{t}^{\prime}, J_{t}^{\prime}\right)$ and to use it to define a chain morphism $F C_{\bullet}\left(H_{t}, J\right) \rightarrow F C_{\bullet}\left(H_{t}^{\prime}, J^{\prime}\right)$ which induces an isomorphism in homology. More precisely, the homotopy $\Psi$ is used to define moduli spaces 
$\mathcal{M}_{\Psi}(x, y)$ for $x \in \phi_{1}^{H_{t}}(L) \cap L$ and $y \in \phi_{1}^{H_{t}^{\prime}}(L) \cap L$. The space $\mathcal{M}_{\Psi}(x, y)$ is defined as follows:

$$
\left\{\begin{array}{l|l}
v: \mathbb{R} \times[0,1] \rightarrow M & \begin{array}{l}
\frac{\partial v}{\partial s}+J_{s} \frac{\partial v}{\partial t}=0, \\
v(s, 0) \in L_{0} v(s, 1) \in \phi_{1}^{H_{s}}\left(L_{0}\right), \\
\lim _{s \rightarrow-\infty} v(s, t)=x(t), \lim _{s \rightarrow+\infty} v(s, t)=y(t)
\end{array}
\end{array}\right\}
$$

For a generic choice of $\Psi$ these spaces are actually finite-dimensional manifolds, and the morphism between the two complexes is defined by counting the number of elements $(\bmod 2)$ of their 0-dimensional components. The latter are proved to be finite by using a compactness result, analogue to 2.3. The same result shows that the 1-dimensional component of $\mathcal{M}(x, y)$ can be completed to a compact boundary manifold such that the parity of the number of boundary points is equivalent to the fact that the morphism defined by $\Psi$ commutes with the Floer differentials.

The same argument as before can be used to get the invariance of the homology of $C_{\bullet}^{\bar{L}}$. Given two collections of points and paths $(\mathcal{C}, \Gamma)$ and $\left(\mathcal{C}^{\prime}, \Gamma^{\prime}\right)$, as above, the paths $v(s, 0) \in \Gamma_{\Psi}$ defined by elements $v$ belonging to 0 -dimensional components of $\mathcal{M}_{\Psi}(x, y)$ define a morphism between the associated lifted complexes $C_{\bullet}^{\bar{L}}$ and $\left(C_{\bullet}^{\bar{L}}\right)^{\prime}$. Claiming that it is a chain morphism is equivalent to the claim that the number of broken paths in $\Gamma_{\Psi}$ admitting lifts which join fixed points $x_{i}$ and $y_{j}$ is even. As above, this is a consequence of Lemma 2.8, adapted to this new setting.

Finally, the arguments of [17] which show that the morphism defined by $\Psi$ between the two usual Floer complexes induces an isomorphism at the homology level, can be used together with 2.8 in the same way, in order to show that the homology of the lifted complex $C_{\bullet}^{\bar{L}}$ does not depend on $\left(H_{t}, J\right)$ either. We will denote this homology by $F H^{\bar{L}}(L)$.

4. Computation of $F H^{\bar{L}}(L)$. When the Lagrangian $L$ is weakly exact its Floer homology is isomorphic to the singular homology of $L$ [18]. To prove this, one has to consider a Morse function $f: L \rightarrow \mathbb{R}$ and a particular Hamiltonian isotopy $\phi_{t}$ which maps $L$ into the graph $L_{t f} \subset U(L) \subset M$, so that the Lagrangian intersections correspond to the critical points of $f$. The notation is that from $\S 1.3$, in particular $U(L)$ is a Weinstein tubular neighborhood of $L$. If $f$ is sufficiently $C^{1}$-small one proves that all the holomorphic strips lie in $U(L)$; the contrary would imply - via Gromov compactness - the existence of a holomorphic disk with boundary in $L$ which is impossible for a weakly exact Lagrangian [34]. For a well-chosen almost complex structure the canonical projection $U(L) \rightarrow L$ maps the holomorphic strips onto the flow lines of a gradient vector field of $f$ with respect to a generic Riemannian metric on $L$. Moreover, this projection defines a one-to-one correspondence between the isolated holomorphic strips and the gradient lines joining critical points of consecutive indices. This means that for these particular choices (which still satisfy the generity 
assumptions required to define Floer homology), the Floer complex is identical to a Morse complex and the result follows.

The latter property of the holomorphic strips above shows that in this particular case the collection $(\mathcal{C}, \Gamma)$ is the one defined by the critical points of $f$ and the isolated gradient lines which join them. So the lifted complex $C_{\bullet}^{\bar{L}}$ coincides with the lifted Morse complex on $\bar{L}$. The homology of the latter is the singular homology of $\bar{L}$. To see this, recall that the stable manifolds of the gradient vector field associated to a Morse function yield a CW-decomposition of $L$ whose lift to $\bar{L}$ computes the homology of this covering space. Therefore we infer:

$$
F H^{\bar{L}}(L) \approx H_{*}(\bar{L}, \mathbb{Z} / 2) .
$$

In the monotone case, when one chooses the same particular Hamiltonian isotopy and almost complex structure, it is no longer true that the holomorphic strips lie in a Weinstein neighborhood $U(L)$. But, as Y.-G. Oh pointed out in [34], the holomorphic strips which lie in $U(L)$ still project onto the gradient lines and the isolated ones are in bijective correspondence to the gradient lines defining the Morse complex. On the other hand, according to the same paper [34] an isolated holomorphic strip of finite energy which leaves $U(L)$ connects two critical points $x, y$ of $f$ which satisfy

$$
\operatorname{Ind}(x)-\operatorname{Ind}(y)=1-l N_{L},
$$

for some positive integer $l$.

In this particular case, the Floer complex can be graded by the Morse index. Therefore, given an integer $l$, the count $(\bmod 2)$ of the isolated holomorphic strips satisfying the index relation above defines for each integer $k$ a map

$$
\partial_{l}: F C_{k} \rightarrow F C_{k-1+l N_{L}} .
$$

Of course, $\partial_{l}$ vanishes for $l>\left[\frac{\operatorname{dim}(L)+1}{N_{L}}\right]$. The Floer differential $\partial: F C \bullet \rightarrow F C \bullet$ writes

$$
\partial=\partial_{0}+\partial_{1}+\cdots+\partial_{l}+\cdots .
$$

Here $\partial_{0}$ is the Morse differential defined by the (projections on $L$ of the) homolorphic strips which do not leave $U(L)$.

From these data Y.-G. Oh [34] and P. Biran [7] inferred the existence of a spectral sequence which converges towards the Floer homology and whose first page is built using the usual (Morse) homology of $L$.

Now, by definition, it is obvious that the differential $\partial^{\bar{L}}$ of the lifted complex $C_{\bullet}^{\bar{L}}$ satisfies to the same properties, namely:

1. It decomposes into a finite sum $\partial^{\bar{L}}=\partial_{0}^{\bar{L}}+\partial_{1}^{\bar{L}}+\cdots$.

2. For the grading given by the Morse index we have $\partial_{l}^{\bar{L}}: C_{k}^{\bar{L}} \rightarrow C_{k-1+l N_{L}}^{\bar{L}}$.

3. The complex $\left(C_{\bullet}^{\bar{L}}, \partial_{0}^{\bar{L}}\right)$ is identical to the lift to $\bar{L}$ of a Morse complex on $L$. 
These properties are sufficient for the existence of a spectral sequence analogous to the one defined by Biran and Oh. The precise statement is:

Theorem 2.9. Denote by $A$ the subring $\mathbb{Z} / 2\left[T^{N_{L}}, T^{-N_{L}}\right]$ of the Laurent polynomials with $\mathbb{Z} / 2$-coefficients and by $A^{k N_{L}} \subset A$ the subgroup $\mathbb{Z} / 2 \cdot T^{k N_{L}}$, for any integer $k$. There exists a spectral sequence $\left\{E_{r}^{p, q}, d_{r}\right\}$ which converges to the lifted Floer homology $\mathrm{FH}^{\bar{L}}(\mathrm{~L})$ and which satisfies the following properties (all the tensor products below are over $\mathbb{Z} / 2)$ :

- $E_{0}^{p, q}=C_{p+q-p N_{L}}^{\bar{L}} \otimes A^{p N_{L}}, d_{0}=\left[\partial_{0}^{\bar{L}}\right] \otimes \mathrm{Id}$.

- $E_{1}^{p, q}=H_{p+q-p N_{L}}(\bar{L}, \mathbb{Z} / 2) \otimes A^{p N_{L}}, d_{1}=\left[\partial_{1}^{\bar{L}}\right] \otimes T^{-N_{L}}(\cdot)$, where

$$
\left[\partial_{1}^{\bar{L}}\right]: H_{p+q-p N_{L}}(\bar{L}, \mathbb{Z} / 2) \rightarrow H_{p+q-1-(p-1) N_{L}}(\bar{L}, \mathbb{Z} / 2)
$$

is induced by $\partial_{1}^{\bar{L}}$.

- For every $r \geq 1, E_{r}^{p, q}$ has the form $E_{r}^{p, q}=V_{r}^{p, q} \otimes A^{p N_{L}}$ with $d_{r}=\delta_{r} \otimes$ $T^{-r N_{L}}$, where $V_{r}^{p, q}$ are vector spaces over $\mathbb{Z} / 2$ and $\delta_{r}: V_{r}^{p, q} \rightarrow V_{r}^{p-r, q+r-1}$ are homomorphisms defined for every $p, q$ and satisfying $\delta_{r} \circ \delta_{r}=0$. Moreover:

$$
V_{r+1}^{p, q}=\frac{\operatorname{Ker}\left(\delta_{r}: V_{r}^{p, q} \rightarrow V_{r}^{p-r, q+r-1}\right)}{\operatorname{Im}\left(\delta_{r}: V_{r}^{p+r, q-r+1} \rightarrow V_{r}^{p, q}\right)},
$$

and for $r=0,1$ we have $V_{0}^{p, q}=C_{p+q-p N_{L}}^{\bar{L}}, V_{1}^{p, q}=H_{p+q-p N_{L}}(\bar{L}, \mathbb{Z} / 2)$, $\delta_{1}=\left[\partial_{1}^{\bar{L}}\right]$.

- The spectral sequence collapses at page $\left[\frac{\operatorname{dim}(L)+1}{N_{L}}\right]+1$ and for all $p \in \mathbb{Z}$, $\bigoplus_{q \in \mathbb{Z}} E_{\infty}^{p, q} \approx F H^{\bar{L}}(L)$.

The proof of 2.9 is analogous to the proof of Theorem 5.2.A in [7]. It is purely algebraic, and it applies to any graded complex whose differential (in our case $\partial^{\bar{L}}$ ) satisfies to the conditions 1-3 above. The spectral sequence $\left\{E_{r}^{p, q}\right\}$ is associated to an increasing filtration of the complex $\widetilde{C}_{\bullet}$ :

$$
\widetilde{C}_{l}=\bigoplus_{k \in \mathbb{Z}} C_{l-k N_{L}}^{\bar{L}} \otimes A^{k N_{L}},
$$

which is endowed with the differential

$$
\tilde{d}=\partial_{0} \otimes \mathrm{Id}+\partial_{1} \otimes T^{-N_{L}}(\cdot)+\partial_{2} \otimes T^{-2 N_{L}}(\cdot)+\cdots .
$$

As in [7], one can show that the homology of this complex is isomorphic to the Floer homology $F H^{\bar{L}}(L)$. 
Remark that the above theorem immediately implies 2.1 for $L$ monotone. Indeed, if $\delta_{1}=0$, then, according to $2.9, V_{2}^{p, q}=V_{1}^{p, q}=H_{p+q-p N_{L}}(\bar{L}, \mathbb{Z} / 2)$, and

$$
\delta_{2}: H_{p+q-p N_{L}}(\bar{L}, \mathbb{Z} / 2) \rightarrow H_{p+q-1-(p-1) N_{L}}(\bar{L}, \mathbb{Z} / 2) .
$$

Actually, the proof of [7] shows that $\delta_{2}=\left[\partial_{2}^{\bar{L}}\right]$. Analogously, if $\delta_{1}=\delta_{2}=\cdots=$ $\delta_{l-1}=0$, then $\delta_{l}$ is defined on the homology of $\bar{L}$ (by $\left[\partial_{l}^{\bar{L}}\right]$ ) and its degree is $-1+l N_{L}$. Finally, if all the $\delta_{i}$ 's vanish then the spectral sequence $E_{r}^{p, q}$ collapses at page 1 and therefore, applying again 2.9 , we have

$$
F H^{\bar{L}}(L) \approx H_{*}(\bar{L}, \mathbb{Z} / 2) .
$$

This finishes the proof of 2.1 for homologies with $\mathbb{Z} / 2$-coefficients.

5. Change of coefficients. A Lagrangian submanifold $L \subset M$ is called relatively spin if there exists a class $s t \in H^{2}(M, \mathbb{Z} / 2)$ that restricts to the second Stiefel-Whitney class $w_{2}(L)$ of $L$. For such submanifolds, also supposed to be orientable, it was proved in [22] that the spaces of holomorphic strips $\mathcal{M}(x, y)$ can be oriented and that under this hypothesis the Floer complex $F C$ • can be defined over $\mathbb{Z}$-coefficients.

In our case, when $L$ is orientable and relatively spin, the lifted complex $C_{\bullet}^{\bar{L}}$ is constructed using a collection of oriented paths $\Gamma$, which enables us to define it over $\mathbb{Z}$. To show that it is a $\mathbb{Z}$-complex whose homology only depends on $L \subset M$ and on the chosen covering space $\bar{L}$ one can use the same proof as above.

If we chose the universal cover $\widetilde{L}$ as covering space, then $C_{\bullet}^{\widetilde{L}}$, can be seen as a free, finite-dimensional complex over $\mathbb{Z} / 2\left[\pi_{1}(L)\right]$ (resp. over $\mathbb{Z}\left[\pi_{1}(L)\right]$ when $L$ is orientable and relatively spin). Of course we can change the coefficients by tensoring with any $\mathbb{Z} / 2\left[\pi_{1}(L)\right]$-module $R$, for instance with the Novikov ring associated to some morphism $u: \pi_{1}(L) \rightarrow \mathbb{Z}$.

In all these situations the homology of the complex $C_{\bullet}^{\bar{L}}$ is related in the same manner as for $\mathbb{Z} / 2$-coefficients to the homology of $\bar{L}$ with coefficients in the new coefficient ring. In particular, in the case we have just considered, the latter is the Novikov homology $H_{*}(L, u)$ (for definition and related properties, see for instance [13], [14]).

The proof of 2.1 is now complete.

\section{Applications}

In this section we prove our main results which we stated in $\S 1$ and other applications of 2.1 .

3.1. Aspherical Lagrangian submanifolds. Proof of Theorems 1.5 and 1.6. The idea of the proofs is that, under the given hypothesis, the spectral sequence given by 
2.1 collapses at page 1, which means that the lifted Floer homology associated to the universal cover of $L$ is isomorphic to the singular homology of $\widetilde{L}$. On the other hand, since $L$ is displaceable through a Hamiltonian isotopy, the lifted Floer homology vanishes. This is contradictory and therefore Theorem 2.1 should not apply here. The only possible reason for that is the fact that the Maslov number $N_{L}$ is less than 3 .

Proof of 1.5. a) If $N_{L} \geq 3$ then we get the applications $\delta_{i}$ provided by 2.1. But since $L$ is aspherical $H_{i}(\widetilde{L})=0$ for $i \neq 0$, which implies that $\delta_{i}=0$ for all $i$. Therefore, according to 2.1 ,

$$
H\left(C_{\bullet}^{\tilde{L}}\right) \approx H(\tilde{L}, \mathbb{Z} / 2) .
$$

Since $L$ is displaceable, the left term vanishes, whereas the right term is not zero in degree $i=0$. This contradiction implies $N_{L} \leq 2$.

We use the following well-known result [2] (see also [21], Lemma 2.5):

Proposition 3.1. If $L$ is orientable then $N_{L}$ is even. The converse is true if $\pi_{1}(M)$ is trivial.

The conclusion of 1.5 follows.

b) Since $L$ is orientable, its Maslov number $N_{L}$ is even and therefore all the applications $\delta_{i}$ provided by 2.1 have an odd degree. Suppose $N_{L} \geq 3$. The homology of $\widetilde{L}$ is zero in odd degrees. This implies $\delta_{1}=0$ and $E_{2}^{p, q}=E_{1}^{p, q}$ in the spectral sequence of 2.9. The same argument shows that all the applications $\delta_{i}$ vanish, which implies that the singular homology of $\widetilde{L}$ is zero. But this is impossible and therefore $N_{L}=2$.

c) Denote by $\mathcal{A}_{\text {reg }}$ the generic set of compatible almost complex structures for which the usual Floer complex $\left(F C_{\bullet}, \partial\right)$ is defined. Consider $J \in \mathcal{G}_{\text {reg }}$ and denote

$$
\mathcal{M}(M, J ; 2)=\left\{w:(D, \partial D) \rightarrow(M, L) \mid \bar{\partial}_{J} w=0, \mu(w)=2\right\} .
$$

By standard transversality results ([16], see also [10], Chapter 3) one gets that for generic $J, \mathcal{M}(M, J ; 2)$ is a manifold of dimension $n+2$. It is important to notice here that a crucial point in the proof of the transversality is the fact that all the disks in $\mathcal{M}(M, J ; 2)$ are simple. This is a consequence of the monotonicity of $L$ and of a result of D. Kwon and Y.-G. Oh [28] and L. Lazzarini [30] (see again [10]). The monotonicity of $L$ also implies that $\mathcal{M}(M, J ; 2)$ is closed. Indeed all the holomorphic disks of this manifold have the same area, so Gromov's compactness [26] applies. On the other hand, since $L$ is monotone and the disks have minimal Maslov number, no bubbling can occur.

The unparametrized $J$-holomorphic disks of Maslov number 2 passing through a given point $p \in L$ can be identified with the preimage $\mathrm{ev}^{-1}(p)$ of an evaluation map

$$
\text { ev }: \mathcal{N} \rightarrow L
$$


where $\mathcal{N}=\left(\mathcal{M}(M, J ; 2) \times S^{1}\right) / \operatorname{PSL}(2, \mathbb{R})$ and $\operatorname{PSL}(2, \mathbb{R})=\operatorname{Aut}(D)$ acts on $\left(\mathcal{M}(M, J ; 2) \times S^{1}\right)$ by

$$
h \cdot(w, z)=\left(w \circ h, h^{-1}(z)\right)
$$

The evaluation map is given by $\operatorname{ev}([w, z])=w(z)$, for $[w, z] \in \mathcal{N}$.

The closed manifold $\mathcal{N}$ is $n$-dimensional and in particular for a generic $p$, the preimage $\mathrm{ev}^{-1}(p)$ is finite. Following the notation of [33], denote by $\Phi_{L}(p)$ the number of elements of $\mathrm{ev}^{-1}(p)$, modulo 2 . This number does not depend on the choice of the regular value $p$ : it is the mod-2 degree of the evaluation map. Y.-G. Oh shows in [33] that in order to define the Floer homology $F H\left(L_{0}, L_{1}\right)$ in the case where $N_{L_{i}}=2$ one needs the hypothesis $\Phi_{L_{0}}+\Phi_{L_{1}}=0$. This comes from the fact that the 2-dimensional component of the trajectory spaces $\mathcal{M}(x, y)$ can be compactified by adding the broken trajectories and the holomorphic disks of Maslov index 2 with boundary in one of the $L_{i}$ 's, passing through the intersection points; the latter occur as bubbles of sequences in $\mathcal{M}(x, y)$ (when $x=y$ ). If their number is even then the relation $\partial^{2}=0$ is still valid.

Note that Y.-G. Oh also shows:

Theorem 3.2. When $L_{1}$ is a Hamiltonian deformation of $L_{0}$ then the above relation

$$
\Phi_{L_{0}}+\Phi_{L_{1}}=0
$$

is satisfied and therefore the Floer homology $F H(L)$ can be defined.

But for the definition of the lifted Floer homology $F H^{\widetilde{L}}(L)$ this is no longer sufficient as can be seen in the example of two circles in $\mathbb{C}$ intersecting in two points which we explained in $\$ 1.3 .2$. Recall that the lifted Floer complex was defined (for $N_{L} \geq 3$ ) using the paths $w(s, 0)$ defined by the isolated holomorphic strips of $\mathcal{M}(x, y)$. To define it for $N_{L}=2$ (with $\mathbb{Z} / 2$-coefficients) one needs for any $x \in L \cap L_{1}, L_{1}=\phi_{1}(L)$ and for any homotopy class $g \in \pi_{1}(L)$ an even number of broken isolated trajectories $w$ from $x$ to $x$ whose associated paths $w(s, 0) \subset L$ define a loop in the class $g$. This is clearly not true in the case of the two circles, as the two broken paths lie in different homotopy classes.

In the general situation, we claim that the Floer homology $F H^{\widetilde{L}}(L)$ can be defined if for any $g \in \pi_{1}(L)$ the number of $J$-holomorphic disks passing through a generic $p \in L$ and whose boundary realize $g$ is even. The same arguments as above (fixing the homotopy class of the boundary in the definitions of $\mathcal{M}, \mathcal{N}$ ) show that the parity of this number does not depend on the generic choice of $p$. Denote it by $\Phi_{g, L} \in\{0,1\}$; clearly

$$
\Phi_{L}=\sum_{g \in \pi_{1}(L)} \Phi_{g, L} \bmod 2
$$


Let us proof our claim. As explained above, it can happen that in order to compactify a 1-dimensional connected component of $\mathscr{L}^{1}(x, x)$ (where $\left.x \in L \cap \phi_{1}(L)\right)$, one has to add a broken trajectory and a holomorphic disk $D$ with boundary in $L$ or $\phi_{1}(L)$. The homotopy class of the boundary $\partial D \subset L$ is determined by the paths $w(s, 0)$ defined by the holomorphic strips corresponding to the elements in $\mathscr{L}^{1}(x, x)$. When the boundary contains a holomorphic disk $(D, \partial D) \subset\left(M, L_{1}\right)$, then the loops defined in $L$ by the trajectories of $\mathscr{L}(x, x)$ are necessarily contractible in $L$. Therefore, counting modulo 2 , we have $\Phi_{g, L}$ broken trajectories in the class $g$ when $g \neq 0$ and $\Phi_{0, L}+\Phi_{L_{1}}$ for $g=0$. Using the fact that $\Phi_{L_{1}}=\Phi_{L}$ and the relation (1) we infer that the lifted Floer complex is defined provided that $\Phi_{g, L}=0$ for any $g \in \pi_{1}(L), g \neq 0$.

Now we are able to finish our proof. If for any non-zero $g \in \pi_{1}(L)$ there is some $p \in L$ such that there is no holomorphic disk with boundary in the class $g$, passing through $p$, then $\Phi_{g, L}=0$ for any $g \neq 0$, so $F H^{\widetilde{L}}(L)$ is defined. But this leads to a contradiction like in the proof of 1.5.a). So, the proof is finished for $J \in \mathcal{g}_{\text {reg. }}$. For an arbitrary $J$, take a sequence $J_{n} \in \mathcal{J}_{\text {reg }}$ which converges towards $J$. Fix $p \in L$ and consider $J_{n}$-holomorphic disks $w_{n}:(D, \partial D) \rightarrow(M, L)$ such that $\mu\left(w_{n}\right)=2$ and $p \in w_{n}(\partial D)$. Using again Gromov's compactness [26] we find that $w_{n}$ converges towards a $J$-holomorphic disk whose boundary passes through $p$. There is no bubbling here because of the monotonicity of $L$ and the fact that the Maslov index is minimal. The boundary of the limit is not trivial in $\pi_{1}(L)$ by an argument which is similar to the one in the proof of 2.8 (see [15], Lemma 3.16).

The proof of 1.5 is now complete.

Using a quite similar argument one can prove the following version of 1.5:

Theorem 3.3. Let $M$ be a monotone symplectic manifold which has the property that any compact subset is displaceable through a Hamiltonian isotopy and let $L \subset M$ be a monotone Lagrangian submanifold.

a) If for some integer $k \geq 1$ we have $H_{i}(\tilde{L}, \mathbb{Z} / 2)=0$ for $i>k$, then

$$
N_{L} \in[1, k+1] \text {. }
$$

b) Suppose that $L$ is orientable. If $H_{*}(\tilde{L}, \mathbb{Z} / 2)$ is of finite dimension over $\mathbb{Z} / 2$ and the Euler characteristic

$$
\chi=\sum_{i=0}^{n}(-1)^{i} \operatorname{dim}\left(H_{i}(\tilde{L}, \mathbb{Z} / 2)\right)
$$

does not vanish, then $N_{L}=2$. Moreover, for any almost complex structure $J$ which is compatible with the symplectic form we have that through every $p \in L$ passes at least a J-holomorphic disk $w:(D, \partial D) \rightarrow(M, L)$ whose Maslov index equals 2 and whose boundary is not trivial in $\pi_{1}(L)$. 
Proof. a) If $N_{L} \geq k+2 \geq 3$, the lifted Floer homology $F H^{\widetilde{L}}$ is well defined. Since the degree of the applications $\delta_{i}$ is greater than or equal to $-1+N_{L} \geq k+1$, all these application vanish and therefore $F H^{\tilde{L}}$ is isomorphic to the singular homology of $\widetilde{L}$. On the other hand $F H^{\widetilde{L}}=0$, as $L$ is displaceable, which is absurd.

b) To prove this statement one has to look at the proof of Theorem 5.2.A in [7]. It is shown that the vector spaces $V_{r}^{p, q}$ in the statement of 2.9 satisfy $V_{r}^{p+1, q}=$ $V_{r}^{p, q+1-N_{L}}$, and the applications $\delta_{r}^{p, q}$ have the same property. Therefore, for $p$, $q$ fixed, $\delta_{r}$ is a differential on the complex $\left(V_{r}^{p, q+k\left(1-r N_{L}\right)}\right)_{k \in \mathbb{Z}}$. This complex is finite (since this assertion is true for $r=1)$ and its homology is $\left(V_{r+1}^{p, q+k\left(1-r N_{L}\right)}\right)_{k}$, according to 2.9. Now fix $p \in \mathbb{Z}$ and consider the Euler characteristic:

$$
\chi_{r}=\sum_{q \in \mathbb{Z}}(-1)^{q} \operatorname{dim}\left(V_{r}^{p, q}\right) .
$$

We show that $\chi_{r}$ does not depend on $r$. Fix a negative odd number $m$. It is quite clear that

$$
\chi_{r}=\sum_{l=m+1}^{0}(-1)^{l} \sum_{k \in \mathbb{Z}}(-1)^{k} \operatorname{dim}\left(V_{r}^{p, l+k m}\right) .
$$

Indeed we have just changed the order of the summands in the writing of $\chi_{r}$. Applying this for $m=1-r N_{L}$ (which is odd, since $L$ is orientable, by 3.1), we get

$$
\chi_{r}=\sum_{q=2-r N_{L}}^{0}(-1)^{q} \chi_{r}^{q}
$$

where $\chi_{r}^{q}$ is the Euler characteristic of the complex $\left(V_{r}^{p, q+k\left(1-r N_{L}\right)}\right)_{k}$. The Euler characteristic of the homology is the same, so one can write

$$
\chi_{r}^{q}=\sum_{k \in \mathbb{Z}}(-1)^{k} \operatorname{dim}\left(V_{r+1}^{p, q+k\left(1-r N_{L}\right)}\right) .
$$

Therefore we get

$$
\chi_{r}=\sum_{q=2-r N_{L}}^{0}(-1)^{q} \sum_{k \in \mathbb{Z}}(-1)^{k} \operatorname{dim}\left(V_{r+1}^{p, q+k\left(1-r N_{L}\right)}\right)=\chi_{r+1} .
$$

The latter equality is obtained by applying the property above for $m=1-r N_{L}$ and $r+1$ instead of $r$.

So $\chi_{r}$ is independent of $r$. On the other hand, according to 2.9, we have

$$
\chi_{1}=\chi\left(H_{*}(\tilde{L}, \mathbb{Z} / 2)\right) \neq 0
$$


and since the spectral sequence collapses and its limit is zero we also have $\chi_{r}=0$ for $r$ sufficiently large. Therefore the lifted Floer complex can not be defined, which means that $N_{L}=2$.

The proof of the existence of a $J$-holomorphic disk of Maslov index 2 passing through a given $p \in L$ is similar to the proof of 1.5.a).

Proof of 1.6. We use the following result of P. Biran ([7], Proposition 4.1.A):

Proposition 3.4. If $L \subset \mathbb{C} \mathbf{P}^{n} \times W$ is Lagrangian monotone then there is a circle bundle $\Gamma_{L} \rightarrow L$ such that $\Gamma_{L}$ admits a monotone Lagrangian embedding into $\mathbb{C}^{n+1} \times$ $W$ and moreover $N_{\Gamma_{L}}=N_{L}$.

If $L$ is aspherical then it is easy to see that $\Gamma_{L}$ is aspherical, too. Since it is displaceable through a Hamiltonian isotopy, the result follows by 1.5 .

\subsection{Lagrangian submanifolds with maximal Maslov number. Proof of Theo- rems 1.7 and 1.8 .}

Proof of Theorem 1.7. a) As $N_{L}=n+1 \geq 3$ the lifted Floer complex is well defined. We know that its homology $F H^{\bar{L}}(L)$ vanishes because $L$ is displaceable. On the other hand, we know by 2.9 that the spectral sequence $\left\{E_{r}^{p, q}\right\}$ which converges towards this homology, collapses at page 2 . So, according to 2.9 we have

$$
0=E_{2}^{p, q}=\frac{\operatorname{Ker}\left(\left[\partial_{1}^{\bar{L}}\right]: E_{1}^{p, q} \rightarrow E_{1}^{p-1, q}\right)}{\operatorname{Im}\left(\left[\partial_{1}^{\bar{L}}\right]: E_{1}^{p+1, q} \rightarrow E_{1}^{p, q}\right)},
$$

which for $q=p-p N_{L}+i$ gives

$$
0=E_{2}^{p, p-p N_{L}+i}=\frac{\operatorname{Ker}\left(\left[\partial_{1}^{\bar{L}}\right]: H_{i}(\bar{L}, \mathbb{Z} / 2) \rightarrow H_{n+i}(\bar{L}, \mathbb{Z} / 2)\right)}{\operatorname{Im}\left(\left[\partial_{1}^{\bar{L}}\right]: H_{i-n}(\bar{L}, \mathbb{Z} / 2) \rightarrow H_{i}(\bar{L}, \mathbb{Z} / 2)\right)}
$$

Applying this equality for $i=1, \ldots, n-1$ we find that $\bar{L}$ is a 2-homology sphere for any covering space $\bar{L}$. In particular, for $\bar{L}=L$ we have $H^{i}(L, \mathbb{Z} / 2)=0$, for $i=1,2$ and therefore we infer that $L$ is spin. $\operatorname{So~} \operatorname{dim}(L)=N_{L}-1$ is odd (by 3.1 , since $L$ is oriented) and the whole theory works for $\mathbb{Z}$-coefficients. The same argument then shows that any covering $\bar{L}$ is a $\mathbb{Z}$-homology sphere. Let us prove that $L$ is also simply connected. If not, take a non-zero element $g \in \pi_{1}(L)$ and consider the Abelian subgroup $G=\langle g\rangle$ and the associated covering space $\bar{L}$. Then $H_{1}(\bar{L}, \mathbb{Z})=G$ which contradicts the fact that $\bar{L}$ is a $\mathbb{Z}$-homology sphere. Finally, using the (proofs of the) Poincaré conjecture, (S. Smale, M. Freedman, G. Perelman) we infer that $L$ is homeomorphic to $S^{n}$, as claimed. 
b) As above, the lifted Floer complex is defined, its homology vanishes, and the spectral sequence converging to it collapses at page 2 . Consider an arbitrary covering $\bar{L} \rightarrow L$. We get, as in the proof of a) for $i=1, \ldots, n$ :

$$
E_{2}^{p, p-p N_{L}+i}=\frac{\operatorname{Ker}\left(\left[\partial_{1}^{\bar{L}}\right]: H_{i}(\bar{L}, \mathbb{Z} / 2) \rightarrow H_{i+n-1}(\bar{L}, \mathbb{Z} / 2)\right)}{\operatorname{Im}\left(\left[\partial_{1}^{\bar{L}}\right]: H_{-n+i+1}(\bar{L}, \mathbb{Z} / 2) \rightarrow H_{i}(\bar{L}, \mathbb{Z} / 2)\right)} .
$$

We infer that

$$
H_{i}(\bar{L}, \mathbb{Z} / 2)=0
$$

for $i=2, \ldots, n-2$ and

$$
\begin{aligned}
& {\left[\partial_{1}^{\bar{L}}\right]: H_{1}(\bar{L}, \mathbb{Z} / 2) \approx H_{n}(\bar{L}, \mathbb{Z} / 2),} \\
& {\left[\partial_{1}^{\bar{L}}\right]: H_{0}(\bar{L}, \mathbb{Z} / 2) \approx H_{n-1}(\bar{L}, \mathbb{Z} / 2) .}
\end{aligned}
$$

Suppose first that $L$ is not orientable. Choosing $\bar{L}=\widetilde{L}$ we get $H_{n}(\widetilde{L})=0$, so $\widetilde{L}$ is not compact, and therefore $\pi_{1}(L)$ is infinite. We know that $H_{1}(L, \mathbb{Z} / 2) \approx$ $H_{n}(L, \mathbb{Z} / 2)=\mathbb{Z} / 2 Z$. Take an element $g \in \pi_{1}(L)$ which is not in the kernel of the Hurewicz morphism $\pi_{1}(L) \rightarrow H_{1}(L, \mathbb{Z} / 2)$ and consider the covering $\bar{L} \rightarrow$ $L$ associated to the Abelian subgroup $G=\langle g\rangle$. If $g$ is of finite order - which has to be even, the covering $\bar{L} \rightarrow L$ is infinite (since $\pi_{1}(L)$ is infinite), therefore $H_{n}(\bar{L}, \mathbb{Z} / 2)=0$ and $H_{1}(\bar{L}, \mathbb{Z} / 2)=0$, by $(2)$. On the other hand $H_{1}(\bar{L}, \mathbb{Z} / 2)=$ $G \otimes_{\mathbb{Z}} \mathbb{Z} / 2 \neq 0$, as $G$ is a cyclic group $\mathbb{Z} / 2 l$. This contradiction implies that $G$ is an infinite cyclic group. In particular $H_{1}(\bar{L}, \mathbb{Z} / 2) \neq 0$, and by (2) $H_{n}(\bar{L}, \mathbb{Z} / 2) \neq 0$, which means that $\bar{L}$ is compact and $G$ has finite index in $\pi_{1}(L)$, as claimed.

If $M$ is an exact symplectic manifold then 1.2 applies and $H^{1}(L, \mathbb{R}) \neq 0$, a non-zero class being given by the restriction to $L$ of a primitive of the symplectic form. Therefore the first Betti number of $L$ is not zero. Consider a non-vanishing morphism $u: \pi_{1}(L) \rightarrow \mathbb{Z}$ and denote by $K$ its kernel. We show that $K$ is finite of odd order.

Indeed, take an element $t \in \pi_{1}(L)$ such that $u(t)=1$ and consider the covering $\bar{L} \rightarrow L$ corresponding to the infinite cyclic subgroup $G$ spanned by $t$. As above, using the relation (2) we infer that $\bar{L} \rightarrow L$ is a finite covering. It is easy to see that different elements of $K=\operatorname{Ker}(u)$ lie in different classes of the quotient $\pi_{1}(L) / G$ and therefore $K$ is finite. Moreover, if we suppose that an element $g \in K$ has even order, then, taking for $\bar{L}$ the covering associated to $G=\langle g\rangle$, we find as above that $H_{1}(\bar{L}, \mathbb{Z} / 2) \neq 0$, whereas $H_{n}(\bar{L}, \mathbb{Z} / 2)=0$ since the covering is infinite. This contradicts the relation (2).

Let us now consider the case where $L$ is orientable (so $n$ is even). Since it is also spin (by (1)), the relations (1) and (2) are valid for integer coefficients. Taking $\bar{L}=L$, we get $H_{1}(L, \mathbb{Z})=\mathbb{Z}$, which implies $H^{1}(L, \mathbb{Z})=\mathbb{Z}$. Therefore, as above 
there is an exact sequence of groups

$$
0 \rightarrow K \rightarrow \pi_{1}(L) \rightarrow \mathbb{Z} \rightarrow 0
$$

where $K$ is finite. Now, using the same argument as above for the covering $\bar{L}$ associated to $\langle g\rangle$, for an arbitrary $g \in K$, we find that $H_{1}(\bar{L}, \mathbb{Z}) \neq 0$ unless $g$ is the identity. Using the relation (2), this implies that $K=\{1\}$, so $\pi_{1}(L) \approx \mathbb{Z}$, as claimed.

For $\operatorname{dim}(L) \geq 6$, F. Latour and A. Pajitnov independently established an algebraic criterion for the existence of a fibration of $L$ over the circle [29], [35]. For $\pi_{1}(L)=\mathbb{Z}$ we get (see for instance [14]):

Theorem 3.5. If $n=\operatorname{dim}(L) \geq 6$ and $\pi_{1}(L)=\mathbb{Z}$, then there exists a fibration $f: L \rightarrow S^{1}$ if and only if the Novikov homology $H_{*}(L ; u)$ vanishes, where $u=$ $\left[f^{*}(d \theta)\right] \in H^{1}(L, \mathbb{Z})$.

According to 2.1 the relations (1) and (2) are also valid for the Novikov homology with respect to any 1 -cohomology class $u$. On the other hand, for any $u \neq 0$ one can show that $H_{0}(L ; u)=0$ and $H_{n}(L ; u)=0$ (see for instance [13]). Using (1), (2) and (3) we find that for $u=\mathrm{id}_{\mathbb{Z}}, H_{*}(L, u)=0$ and therefore, $L$ admits a fibration over the circle, by 3.5. Denote by $F$ a fiber of this fibration. We know that $\pi_{1}(F)=\operatorname{Ker}(u)$, so $F$ is simply connected. We also have that $\widetilde{L}$ is diffeomorphic to $F \times \mathbb{R}$ and in particular

$$
H_{*}(\tilde{L}, \mathbb{Z}) \approx H_{*}(F, \mathbb{Z})
$$

We infer from (2) that $F$ is a simply connected homology sphere, therefore it is homeomorphic to the standard $(n-1)$-sphere, using the proof of Poincaré's conjecture.

The proof of 1.7 is now complete.

Proof of Theorem 1.8. a) The vanishing of the first homology group of $L$ implies that $L$ is monotone with $N_{L}=2(n+1)$. Applying 3.4 we get a monotone Lagrangian submanifold $\Gamma_{L} \subset \mathbb{C}^{n+1} \times X$ which has the same Maslov number $N_{\Gamma_{L}}=N_{L}=$ $\operatorname{dim}\left(\Gamma_{L}\right)+1$. Moreover $\Gamma_{L} \rightarrow L$ is a circle fibration.

The submanifold $\Gamma_{L}$ satisfies to the hypothesis of Theorem 1.7.b and therefore $\pi_{1}\left(\Gamma_{L}\right) \approx \mathbb{Z}$. Moreover, considering the lifted Floer complex associated to the universal cover $\widetilde{\Gamma}_{L}$, the relations (1), (2) and (3) from the proof of 1.7 imply that $H_{i}\left(\widetilde{\Gamma}_{L}\right)=0$ for $i \neq 0,2 n+1$, and $H_{2 n+1}\left(\widetilde{\Gamma}_{L}\right) \approx \mathbb{Z}$. Therefore, using Hurewicz's isomorphism the first $2 n+1$ homotopy groups of $\Gamma_{L}$ are isomorphic to the corresponding homotopy groups of $S^{2 n+1}$. From the long exact sequence of the fibration $\Gamma_{L} \rightarrow L$ we infer that

$$
\pi_{i}(L) \approx \pi_{i}\left(S^{2 n+1}\right) \text { for } i=2, \ldots, 2 n+1,
$$


and, since $\pi_{1}\left(\Gamma_{L}\right)$ is $\mathbb{Z}, \pi_{1}(L)$ is Abelian. But as $H_{1}(L, \mathbb{Z})=0, L$ is simply connected. Therefore $L$ is homeomorphic to $S^{2 n+1}$ according to (the proof of) the Poincaré conjecture.

b) As above $L$ is monotone with $N_{L}=2(n+1)$. Again, we can apply 3.4 and we get a monotone Lagrangian in $\mathbb{C}^{n+1} \times \mathbb{C} \mathbf{P}^{n}$ which is a circle fibration over $L$ and has the same Maslov number. We can therefore use Theorem1.7.a and infer that $\Gamma_{L}$ is homeomorphic to the $(2 n+1)$-sphere, as claimed. In particular $L$ is simply connected.

c) As pointed out in [7], one can easily see that $L$ is monotone with $N_{L}=n+1$. We consider as above the Lagrangian submanifold $\Gamma_{L} \subset \mathbb{C}^{n+1}$ which has the same Maslov number. We can use 2.1 with integer coefficients because $\Gamma_{L}$ is spin (using again 2.1 with mod-2 coefficients) and orientable (by 3.1, since $N_{\Gamma_{L}}$ is even). As in the proof of a) above we get that $\pi_{1}\left(\Gamma_{L}\right)=\mathbb{Z}$ and the universal cover $\widetilde{\Gamma}_{L}$ has the same homotopy groups $\pi_{i}$ as $S^{n}$, for $i=1, \ldots, n$. Using the long exact sequence of the fibration $\Gamma_{L} \rightarrow L$ we find $\pi_{n}(L)=\mathbb{Z}, \pi_{i}(L)=0$ for $i=3, \ldots, n-1$ and we have an exact sequence

$$
0 \rightarrow \pi_{2}(L) \rightarrow \pi_{1}\left(S^{1}\right)=\mathbb{Z} \rightarrow \pi_{1}\left(\Gamma_{L}\right)=\mathbb{Z} \rightarrow \pi_{1}(L) \rightarrow 0 .
$$

It follows that $\pi_{1}(L)$ is cyclic and, since $H_{1}(L, \mathbb{Z})$ is 2-torsion, it follows that $\pi_{1}(L)=\mathbb{Z} / 2$. From the exact sequence we then infer that $\pi_{2}(L)=0$. So the universal cover of $L$ is a homotopy sphere and therefore it is homeomorphic to the $n$-sphere.

3.3. Lagrangian submanifolds in the cotangent bundle. Proof of Theorem 1.9. Let $K \subset M$ be a Lagrangian submanifold. If $L \subset T^{*} K$ is Lagrangian then by Darboux' Theorem it follows that $L$ admits a Lagrangian embedding into $M$. We need the following result:

Proposition 3.6. Suppose that $K \subset M$ is monotone and that $L \subset T^{*} K$ is exact with vanishing Maslov class. Then $L \subset M$ is also monotone. Moreover, if the morphism $\pi_{1}(L) \rightarrow \pi_{1}(K)$ induced by the projection is surjective, then $N_{L}=N_{K}$.

Proof. Since $L$ is exact it is easy to see that (using the notation of the first section) we have

$$
I_{\omega}^{L \hookrightarrow M}=I_{\omega}^{K \hookrightarrow M} \circ p,
$$

where $p: \pi_{2}(M, L) \rightarrow \pi_{2}\left(M, T^{*} K\right) \approx \pi_{2}(M, K)$ is the canonical morphism.

It is also known and not very hard to prove (see [4], Chapter I, Proposition A.3.3) that when $L$ has a vanishing Maslov class then (again with the notation of §1) we have

$$
I_{\mu}^{L \hookrightarrow M}=I_{\mu}^{K \hookrightarrow M} \circ p .
$$

Therefore $L \subset M$ is monotone. If $\pi_{1}(L) \rightarrow \pi_{1}(K)$ is surjective, then $p$ is also an epimorphism and the conclusion follows. 
Proof of 1.9.a). Let $L \subset T^{*} S^{2 k+1}$ be Lagrangian exact with vanishing Maslov class. As pointed out in $\S 1.2$, the application

$$
z \mapsto([z], \bar{z})
$$

defines a monotone Lagrangian embedding of $S^{2 k+1}$ into $\mathbb{C} \mathbf{P}^{k} \times \mathbb{C}^{k+1}$. It follows by 3.6 that $L$ admits a monotone Lagrangian embedding into $\mathbb{C} \mathbf{P}^{k} \times \mathbb{C}^{k+1}$ of Maslov number $N_{L}=N_{S^{2 k+1}}=2 k+2$. The statement a) of Theorem 1.7 implies the desired result.

Proof of 1.9.b). Let $L \subset T^{*} K$ be a Lagrangian exact submanifold with vanishing Maslov class. There is a finite cover $\bar{L}$ of $L$ which admits an exact Lagrangian embedding into $T^{*} \tilde{K}=T^{*} S^{2 k+1}$ (see [15], Lemma 3.5). Moreover, we have a commutative diagram:

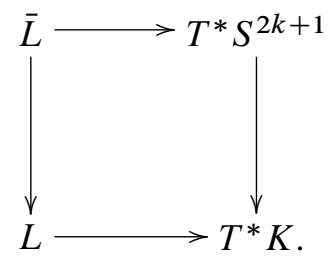

From this diagram one immediately infers that the Maslov class of $\bar{L}$ vanishes. Therefore we can apply the point a) which asserts that $\bar{L}$ is homeomorphic to $S^{2 k+1}$.

When $L=\mathbb{R} \mathbf{P}^{2 k+1}$ we show that $\pi_{1}(L) \rightarrow \pi_{1}\left(\mathbb{R} \mathbf{P}^{2 k+1}\right)$ is an epimorphism. If not, we can lift $L$ to an exact Lagrangian embedding into $T^{*} S^{2 k+1}$, which still has vanishing Maslov class. Using the statement a) we find that $L$ is homeomorphic to a sphere, and in particular $N_{L}=2(2 k+2)$. But this is impossible by the results of P. Biran [7] (There is no Lagrangian sphere in $\left.\mathbb{C} \mathbf{P}^{n}\right)$. So $\pi_{1}(L) \rightarrow \pi_{1}\left(\mathbb{R} \mathbf{P}^{2 k+1}\right)$ is surjective. In this case the mentioned statement of [15] asserts that $\bar{L}=\widetilde{L}$ is a double covering.

Acknowledgements. I thank Alexandru Oancea for our valuable discussions on the subject and Octav Cornea for his useful remarks.

\section{References}

[1] V. I. Arnol'd, First steps in symplectic topology. Russ. Math. Surv. 6 (1986), 3-18. Zbl 0618.58021 MR 0890489

[2] V. I. Arnol'd, On a characteristic class entering the quantizations. Funct. Anal. Appl. 1 (1967), 1-14. Zbl 0175.20303 MR 0211415

[3] M. Audin, Fibrés normaux d'immersions en dimension double, points doubles d'immersions lagrangiennes et plongements totalement réels. Comment. Math. Helv. 63 (1988), 593-623. Zbl 0666.57024 MR 0966952 
[4] M. Audin and J. Lafontaine, eds., Holomorphic curves in symplectic geometry. Introduction: applications of pseudo-holomorphic curves to symplectic topology. Progr. Math. 117, Birkhäuser, Basel 1994, 1-14. Zbl 0802.53001 MR 1274924

[5] M. Audin, F. Lalonde, and L. Polterovich, Symplectic rigidity: Lagrangian submanifolds. In Holomorphic curves in symplectic geometry, ed. by M. Audin and J. Lafontaine, Progr. Math. 117, Birkhäuser, Basel 1994, 271-321. Zbl 0802.53001 MR 1274934

[6] J.-F. Barraud and O. Cornea, Quantization of the Serre spectral sequence. J. Symplectic Geom. 5 (2007), no. 3, 249-280. Zbl 1153.55017 MR 2399677

[7] Paul Biran, Lagrangian non-intersections. Geom. Funct. Anal. 16 (2006), 279-326. Zbl 1099.53054 MR 2231465

[8] P. Biran and K. Cieliebak, Symplectic topology on subcritical manifolds. Comment. Math. Helv. 76 (2001), 712-753. Zbl 1001.53057 MR 1881704

[9] P. Biran and O. Cornea, A Lagrangian quantum homology. In New perspectives and challenges in symplectic field theory, CRM Proc. Lect. Notes 49, Amer. Math. Soc., Providence, RI, 2009, 1-44. Zbl 1185.53087 MR 2555932

[10] P. Biran and O. Cornea, Quantum structures for Lagrangian submanifolds. arXiv:0808.3989v1 [math.SG].

[11] P. Biran and O. Cornea, Rigidity and uniruling for Lagrangian submanifolds. Geom. Topol. 13 (2009), no. 5, 2881-2989. Zbl 1180.53078 MR 2546618

[12] L. Buhovsky, Homology of Lagrangian submanifolds in cotangent bundles. Israel J. Math. 143 (2004), 181-187. Zbl 1090.53063 MR 2106982

[13] M. Damian, Formes fermées non singulières et propriétés de finitude des groupes. Ann. Sci. Ec. Norm. Sup. 33 (2000) no. 3, 301-320. Zbl 0969.57026 MR 1775183

[14] M. Damian, On the homotopy type of finite CW-complexes with polycyclic fundamental group. Trans. Amer. Math. Soc. 361 (2009), no. 4, 1791-1809. Zbl 1179.55005 MR 2465817

[15] M. Damian, Constraints on exact Lagrangians in cotangent bundles of manifolds fibered over the circle. Comment. Math. Helv. 89 (2009), 705-746. Zbl 1190.57017 MR 2534477

[16] D. McDuff and D. Salamon, J-holomorphic curves and symplectic topology. Amer. Math. Soc. Colloq. Publ. 52, Amer. Math. Soc., Providence, RI, 2004. Zbl 1064.53051 MR 2045629

[17] A. Floer, Symplectic fixed points and holomorphic spheres. Comm. Math. Phys. 120 (1989), no. 4, 575-611. Zbl 0755.58022 MR 0987770

[18] A. Floer, Morse theory for Lagrangian intersections. J. Differential Geom. 28 (1988), no. 3, 513-547. Zbl 0674.57027 MR 0965228

[19] A. Floer, The unregularized gradient flow of the symplectic action. Comm. Pure Appl. Math. 41, (1988), no. 6, 775-813. Zbl 0633.53058 MR 0948771

[20] A. Floer, Witten's complex and infinite dimensional Morse theory. J. Differential Geom. 30 (1989), 207-221. Zbl 0678.58012 MR 1001276

[21] K. Fukaya, Application of Floer homology of Langrangian submanifolds to symplectic topology. In Morse theoretic methods in nonlinear analysis and in symplectic topology, ed. by P. Biran and O. Cornea, NATO Sci. Ser. II Math. Phys. Chem. 217, Springer-Verlag, Dordrecht 2006, 231-276. Zbl 1089.53064 MR 2276953 
[22] K. Fukaya, Y.-G. Oh, H. Ohta, and K. Ono, Lagrangian intersection Floer theory: anomaly and obstruction. Part I. AMS/IP Stud. Adv. Math. 46.1, Amer. Math. Soc., Providence, RI; International Press, Somerville, MA, 2009. Zbl 1181.53002 MR 2553465

[23] K. Fukaya, P. Seidel, and I. Smith, Exact Lagrangian submanifolds in simply connected cotangent bundles. Invent. Math. 172 (2008), no. 1, 1-27. Zbl 1140.53036 MR 2385665

[24] K. Fukaya, P. Seidel, and I. Smith, The symplectic geometry of cotangent bundles from a categorical viewpoint. In Homological mirror symmetry, Lecture Notes in Phys. 757, Springer-Verlag, Berlin 2009, 1-26. Zbl 1163.53344 MR 2596633

[25] A. Gadbled, Obstructions to the existence of monotone Lagrangian embeddings into cotangent bundles of manifolds fibered over the circle. Ann. Inst. Fourier (Grenoble) 59 (2009), no. 3, 1135-1175. Zbl 1186.57019 MR 2543665

[26] M. Gromov, Pseudo holomorphic curves in symplectic manifolds. Invent. Math. 82 (1985), 307-347. Zbl 0592.53025 MR 0809718

[27] R. Hind, Lagrangian spheres in $S^{2} \times S^{2}$. Geom. Funct. Anal. 14 (2004), no. 2, 303-318. Zbl 1066.53129 MR 2060197

[28] D. Kwon and Y.-G. Oh, Structure of the image of (pseudo)-holomorphic discs with totally real boundary condition. Comm. Anal. Geom. 8 (2000), no. 1, 31-82. Zbl 0951.32025 MR 1730896

[29] F. Latour, Existence de 1-formes fermées non-singulières dans une classe de cohomologie de de Rham. Inst. Hautes Études Sci. Publ. Math. 80 (1994), 135-194. Zbl 0837.58002 MR 1320607

[30] L. Lazzarini, Decomposition of a J-holomorphic curve. Preprint. http://www.math.jussieu.fr/ lazzarin/articles.html

[31] D. Nadler, Microlocal branes are constructible sheaves. Selecta Math. (N.S.) 15 (2009), no. 4, 563-619. Zbl 1197.53116 MR 2565051

[32] Y.-G. Oh, Floer cohomology of Lagrangian intersections and pseudoholomorphic disks. I. Comm. Pure Appl. Math. 46 (1993), 949-993. Zbl 0795.58019 MR 1223659

[33] Y.-G. Oh, Addendum to: "Floer cohomology of Lagrangian intersections and pseudoholomorphic disks. I." [Comm. Pure Appl. Math. 46 (1993), no. 7, 949-993]. Comm. Pure Appl. Math. bf 48 (1995), no. 11, 1299-1302. Zbl 0847.58036 MR 1367384

[34] Y.-G. Oh, Floer cohomology, spectral sequences, and the Maslov class of Lagrangian embeddings. Internat. Math. Res. Notices 7 (1996), 305-346. Zbl 0858.58017 MR 1389956

[35] A. Pajitnov, Surgery on the Novikov complex. K-theory 10 (1996), 323-412. Zbl 0861.57042 MR 1404410

[36] L. Polterovich, The Maslov class of Lagrange surfaces and Gromov's pseudo-holomorphic curves. Trans. Amer. Math. Soc. 325 (1991), 241-248. Zbl 0719.53016 MR 0992608

[37] L. Polterovich, Monotone lagrangian submanifolds of linear spaces and the Maslov class in cotangent bundles. Math. Z. 207 (1991), 217-222. Zbl 0703.58018 MR 1109663

[38] P. Seidel, Exact Lagrangian submanifolds in $T^{*} S^{n}$ and the graded Kronecker quiver. In Different faces of geometry, Int. Math. Ser. (N.Y.) 3, Kluwer/Plenum, New York 2004, 349-364. Zbl 1070.53049 MR 2103000

[39] C. Viterbo, Intersection de sous-variétés lagrangiennes, fonctionnelles d'action et indice des systèmes hamiltoniens. Bull. Soc. Math. France 115 (1987), no. 3, 361-390. Zbl 0639.58018 MR 0926533 
Received July 11, 2009

Mihai Damian, Université de Strasbourg, IRMA, 7, rue René Descartes, 67084 Strasbourg, France

E-mail: damian@math.u-strasbg.fr 